1. Barker DP, Rutter N. Stress, severity of illness, and outcome in ventilated preterm infants. Arch Dis - Child Fetal Neonatal Ed 1996;75:F187-F190.

2. Krinsley IS. Association between hyperglycemia and inereased hospital mortality in a heterogeneous population-of eritically ill patients. Mayo-Clin Proc 2003;78:1471-8.

3. Srinivasan V, Spinella PC, Drott HR, Roth-CL, Helfaer MA, Nadkarni V. Association of timing, duration, and intensity of hyperglycemia with intensive care unit mortality in critically ill children. Pediatr Crit Care Med 2004;5:329-36.

4. Hays SP, Smith EO, Sunehag AI. Hyperglycemia is a risk factor for early death and morbidity in extremely low birthweight infants. Pediatrics 2006;118:1811-8.

5. van den Berghe $G$, Wouters $P$, Weekers $F$, et al. Intensive in sulin therapy in critically ill patients. N Engl J Med 2001;345: 1359-367.

6. van den Berghe $G$, Wilmer A, Hermans $G$, et al. Intensive insulin therapy in the medical ICU. N Engl J Med 2006;354:449-61.

7. Beardsall K, Vanhaesebrouck S, Ogilvy-Stuart AL, et al. Early insulin therapy in very-low-birth-weight infants. $N$ Engl J Med 2008;359:1873-84.

8. Lucas $\Lambda$, Morley $\mathrm{R}$, Cole TJ. Adverse neurodevelopmental outcome of moderate neonatal hypoglycaemia. BMJ 1988;297: 1304-8.

9. De Vries LS, van Haastert IL, Rademaker KJ, Koopman C, Groenendaal F. Ultrasound abnormalities preceding cerebral palsy in high-risk preterm infants. J Pediatr 2004;144:815-20. 10. Agus MS, Javid PI, Piper HG, et al. The effect of insulin infusion upon protein metabolism in neonates on extracorporeal life support. Ann Surg 2006;244:536-44.

11. Meetze W. Bowsher R, Compton J, Moorehead H. Hyperglyeemia in extremely low birth-weight infants. Biol Neonate 1998; 74:214-21.

12. Collins JW Jr, Hoppe M, Brown K, Edidin DV, Padbury J, Ogata ES. A controlled trial of insulin infusion and parenteral nutrition in extremely low birth weight infants with glucese intolerance. J Pediatr 1991;118:921-7.

13. Hay WW Jr. Intravenous nutrition of the very preterm neonate. Acta Paediatr Suppl 2005;94:47-56.

14. Hey $\mathrm{E}$. Hyperglycaemia and the very preterm baby. Semin Fetal Neonatal Med 2005;10:377-87.

15. Mitanchez D. Glucose regulation in preterm newborn infants. Horm Res 2007;68:265-71.

Copyright (c) 2008 Massachusetts Medical Society.

\title{
Elevated C-Reactive Protein in Atherosclerosis - Chicken or Egg?
}

\author{
Heribert Schunkert, M.D., and Nilesh J. Samani, M.D., F.Med.Sci.
}

One of the most debated topics in cardiovascular medicine is whether $\mathrm{C}$-reactive protein (CRP), a component of the acute-phase response, is a causal factor in the pathogenesis of atherosclerosis. ${ }^{1,2}$ If it is, the implications could be farreaching and include new approaches for the prevention and treatment of myocardial infarction and stroke. ${ }^{3}$ Support for a role of CRP in the pathogenesis of atherosclerosis comes largely from epidemiologic studies that have consistently observed an association between elevated plasma CRP levels and cardiovascular events. ${ }^{1,4,5}$ The statistical strength of such associations is at least as robust as that of established risk factors such as hypertension, diabetes mellitus, and hypercholesterolemia. ${ }^{5}$ However, statistical strength does not imply causality, since confounding factors or reverse causality offer alternative explanations for the association (Fig. 1A). CRP is particularly susceptible to confounding, since multiple cardiovascular risk factors, including smoking, hypertension, obesity, lack of physical activity, and low socioeconomic status, all relate independently to elevated plasma levels of the protein. ${ }^{7,8}$ Reverse causation is also a potential explanation, since atherosclerosis may trigger an elevation of CRP levels.

For established cardiovascular risk factors, causality was proved by randomized treatment trials showing the clinical benefits of lowering blood pressure, cholesterol levels, and glucose levels. As reported in this issue of the Journal, Zacho and coworkers ${ }^{6}$ used a less familiar study design for testing the causality of CRP in atherosclerosis. The instrument these investigators used is genetic variation in the CRP gene that gives rise to variation in plasma CRP levels. They took advantage of the fact that persons are effectively randomly assigned at birth to either higher or lower CRP levels depending on the genetic variant they receive from their parents. ${ }^{9}$ According to Mendel's law of independent assortment, neither endogenous nor exogenous factors disturb this randomization process, which therefore can be termed "mendelian randomization." (This generalization is not entirely free of exceptions, since genes that are very close to one another on a chromosome do not segregate independently; however, it is probably true for most traits.)

Zacho and coworkers investigated the three component associations of a mendelian randomization study (Fig. 1B). First, the effect of CRP genotypes on plasma CRP levels was quantified (Fig. 1B, left line of the triangle). This is relatively easy and can be done with great precision. Second, the magnitude of the association between 


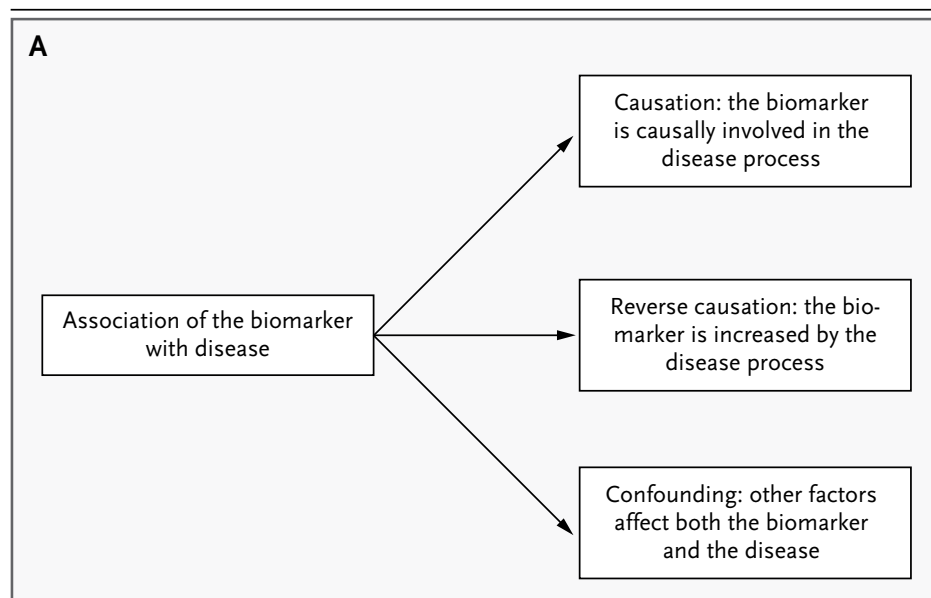

B

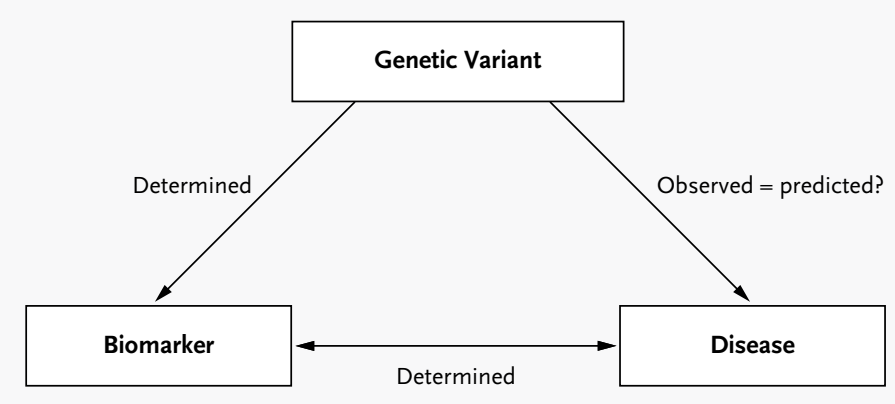

Figure 1. Types of Association of a Biomarker with Disease and the Mendelian Randomization Approach to Identifying a Causal Association.

An association between a biomarker (e.g., C-reactive protein) and disease (e.g., atherosclerotic diseases) may represent a causal relationship (causation), an increase in the biomarker as a consequence of the disease or its treatment (reverse causation), or an association that is spurious because both the biomarker and the disease are affected independently by another known or unknown factor (confounding) (Panel A). The mendelian randomization approach allows determination of a causal relationship between a biomarker and disease (Panel B). It starts with the evidence that the biomarker is associated with the disease (lower line of triangle). The quantitative nature of this association is determined. Genetic variants are then identified that affect the level of the biomarker (left line of triangle), and the quantitative nature of this relationship is also determined. Combining these two sets of information allows prediction of the effect of the genetic variant on disease risk if the association of the biomarker with the disease is causal. This prediction is then tested (right line of the triangle). If the predicted association of the variant with the disease is observed, then this would strongly suggest a causal relationship between the biomarker and the disease (since the relationship between the genetic variant and the disease can go in only one direction). This applies to the association between cholesterol and the risk of coronary artery disease in the study by Zacho et al. ${ }^{6}$ in this issue of the Journal. If, on the other hand, no association is seen between the genetic variant and the disease, as observed for CRP, this would indicate that the association between the biomarker and the disease is due to either reverse causation or confounding. plasma CRP levels and ischemic events (but not the causality of the association) was quantified (Fig. 1B, bottom line of the triangle). Finally, using the information from these two analyses, Zacho et al. predicted the expected effect of the genetic variants on ischemic events (Fig. 1B, right line of the triangle). If the predicted effect and the actually observed effect agreed, one could infer that the relationship between plasma CRP level and the risk of atherosclerotic disease was causal.

The most crucial part in such a study is to obtain a sample size that allows precise estimations of the effect sizes. Zacho et al. measured highsensitivity CRP levels and conducted genotyping for four CRP genetic variants in 50,816 subjects. They found that genetic CRP variants explained a difference in plasma CRP levels of up to $64 \%$ (Fig. 1B, left side). Moreover, the investigators observed the expected association between the plasma CRP level and cardiovascular disease (Fig. 1B, bottom). Despite confirming these two associations, the authors found that none of the CRP variants were associated individually or in combination with the risk of ischemic events (Fig. 1B, right side). Crucially, Zacho et al. were able to reliably estimate the effect on ischemic events that the genetic variants should have had, and they showed that the observed association was markedly different from the predicted effect. As further proof of the validity of their approach, Zacho et al. included a positive control in their experiment: they typed variants in the apolipoprotein E gene, which affect cholesterol level, and found the predicted association between these variants and increased cardiovascular risk.

A positive feature of the study by Zacho et al. is that the various associations were examined in the same population, rather than extrapolated from individual associations observed in different cohorts. A limitation of the study is the partial use of cross-sectional and especially casecontrol cohorts in the analysis, since the use of subjects recruited after the event could introduce a survival bias. The proposed interpretation of the findings relies on the assumption that genetically elevated CRP levels behave similarly to acquired elevations in CRP levels (due to inflammation, for example). It also assumes that lifelong 
genetic elevation of plasma CRP levels does not induce compensatory mechanisms in other systems. Nevertheless, the findings are conclusive and consistent with other mendelian randomization studies of CRP. ${ }^{10-13}$

What are the implications of the findings? First, they strongly indicate that CRP is not causally involved in the pathogenesis of atherosclerotic disease. Thus, immediate targeting of CRP is unlikely to be beneficial in reducing the risk of cardiovascular events. For definitive proof, this hypothesis needs to be tested in randomized clinical trials of CRP inhibitors, but the findings of Zacho et al. and other investigators who have performed genetic studies of $\mathrm{CRP}^{10-13}$ argue against a positive outcome of such studies. However, the findings should not be interpreted as suggesting that factors that cause acquired elevations in CRP levels, such as inflammation, do not play a causal role in atherosclerosis. In fact, the data suggest that confounding factors not considered by the multivariate analysis (such as inflammation) affect the regulation of CRP and the risk of ischemic disease in parallel. Moreover, the study does not diminish the validity of $\mathrm{CRP}$ as a risk marker of atherosclerotic disease. Clinical studies such as the recently terminated Justification for the Use of Statins in Primary Prevention: an Intervention Trial Evaluating Rosuvastatin (known as JUPITER, ClinicalTrials.gov number, NCT00239681) ${ }^{14}$ will establish whether measuring CRP is useful for risk stratification and therapeutic decision making.

From a broader perspective, the study by Zacho et al. provides a landmark example of how genetics may help to illuminate research in the cardiovascular field. Indeed, modern genomic analysis not only may identify new risk genes and thereby mechanisms leading to coronary artery disease ${ }^{15}$ but also may allow the functionality of circulating risk markers to be determined.

No potential conflict of interest relevant to this article was reported.
From the Medizinische Klinik II, Universität zu Lübeck, Lübeck, Germany (H.S.); and the Department of Cardiovascular Sciences, University of Leicester, Leicester, United Kingdom (N.J.S.).

1. Scirica BM, Morrow DA. Is C-reactive protein an innocent bystander or proatherogenic culprit? The verdict is still out. Circulation 2006;113:2128-51.

2. Pepys MB. CRP or not CRP? That is the question. Arterioscler Thromb Vasc Biol 2005;25:1091-4.

3. Pepys MB, Hirschfield GM, Tennent GA, et al. Targeting C-reactive protein for the treatment of cardiovascular disease. Nature 2006;440:1217-21.

4. Danesh J, Wheeler JG, Hirschfield GM, et al. C-reactive protein and other circulating markers of inflammation in the prediction of coronary heart disease. N Engl J Med 2004;350:1387-97. 5. Ridker PM. C-reactive protein and the prediction of cardiovascular events among those at intermediate risk: moving an inflammatory hypothesis toward consensus. J Am Coll Cardiol 2007;49:2129-38.

6. Zacho J, Tybjærg-Hansen A, Jensen JS, et al. Genetically elevated C-reactive protein and ischemic vascular disease. $\mathrm{N}$ Engl J Med 2008;359:1897-908.

7. Everett BM, Kurth T, Buring JE, Ridker PM. The relative strength of C-reactive protein and lipid levels as determinants of ischemic stroke compared with coronary heart disease in women. J Am Coll Cardiol 2006;48:2235-42.

8. Koenig W, Sund M, Fröhlich M, et al. C-reactive protein, a sensitive marker of inflammation, predicts future risk of coronary heart disease in initially healthy middle-aged men: results from the MONICA (Monitoring Trends and Determinants in Cardiovascular Disease) Augsburg Cohort Study, 1984 to 1992. Circulation 1999;99:237-42.

9. Hingorani A, Humphries S. Nature's randomised trials. Lancet 2005;366:1906-8.

10. Timpson NJ, Lawlor DA, Harbord RM, et al. C-reactive protein and its role in metabolic syndrome: mendelian randomisation study. Lancet 2005;366:1954-9.

11. Lange LA, Carlson CS, Hindorff LA, et al. Association of polymorphisms in the CRP gene with circulating C-reactive protein levels and cardiovascular events. JAMA 2006;296:2703-11. 12. Pai JK, Mukamal KJ, Rexrode KM, Rimm EB. C-reactive protein (CRP) gene polymorphisms, CRP levels, and risk of incident coronary heart disease in two nested case-control studies. PLoS ONE 2008;3(1):e1395.

13. Lawlor DA, Harbord RM, Timpson NJ, et al. The association of C-reactive protein and CRP genotype with coronary heart disease: findings from five studies with 4,610 cases amongst 18,637 participants. PLoS ONE 2008;3(8):e3011.

14. Ridker PM, Foncesca FA, Genest J, et al. Baseline characteristics of participants in the JUPITER trial, a randomized placebocontrolled primary prevention trial of statin therapy among individuals with low low-density lipoprotein cholesterol and elevated high-sensitivity C-reactive protein. Am J Cardiol 2007;100:1659-64. 15. Samani NJ, Erdmann J, Hall AS, et al. Genomewide association analysis of coronary artery disease. N Engl J Med 2007;357: 443-53.

Copyright (@) 2008 Massachusetts Medical Society. 